\title{
Multiparticle time-domain analysis of coherent undulator radiation
}

\author{
Vipul Joshi ${ }^{*}$ and Subhendu Ghosh \\ Inter University Accelerator Centre (IUAC), Aruna Asaf Ali Marg, New Delhi 110067, India
}

(Received 30 November 2018; published 19 February 2019)

\begin{abstract}
The physics involving the emission of coherent undulator radiation from a bunch of correlated electrons wiggling inside an undulator has been very well established in the frequency domain. With the aim of describing the process of emission of coherent undulator radiation in time domain while incorporating the effect of hyperbolic field profile of the undulator and various electron beam parameters, we present a timedomain analysis which can serve as a complimentary formulation to the frequency-domain analysis of the coherent undulator radiation. The formulation requires revisiting the equation of motion of an electron moving inside a planar undulator. An electron beam with substantial spread along the direction of the magnetic field will undergo betatron oscillations due to the hyperbolic profile of the undulator fields. We show that the betatron motion can be conveniently expressed in terms of Mathieu functions. The new set of equations of motion have been used to study the effect of betatron motion on the emitted undulator radiation. The single-particle time-domain analysis is then easily extended to multiparticle systems by correctly accounting for the phase-difference between the individual electromagnetic waves emitted by different electrons occupying different phase-space in the bunch.
\end{abstract}

DOI: $10.1103 /$ PhysRevAccelBeams.22.020702

\section{INTRODUCTION}

The interest in studying electromagnetic radiation emitted by energetic charged particles using particle accelerators dates back to the early 1940s. As early as in 1944, Iwanenko and Pomeranchuk showed that the radiative energy loss due to accelerated electrons would impose restrictions on the maximum achievable energy [1]. Although the loss of electron beam energy in form of radiation was considered to be an unwanted process yet Schwinger at General Electric synchrotron facility investigated the properties of synchrotron radiation and developed the formulation for characteristics of the emitted radiation like instantaneous power, angular and spectral distribution etc. by a single electron [2]. A few years later, Ginsburg developed the theory to evaluate coherent and incoherent radiation from oscillating electrons [3]. Soon, the characteristics of the radiation emitted by relativistic particles moving inside periodic array of magnets (undulator) was discussed by Motz and he analytically showed that the coherent radiation in the millimeter regime may be obtained from a few $\mathrm{MeV}$ electrons provided that the bunch length of the electrons is smaller as compared to the

\footnotetext{
*vipuljoshi92@gmail.com
}

Published by the American Physical Society under the terms of the Creative Commons Attribution 4.0 International license. Further distribution of this work must maintain attribution to the author(s) and the published article's title, journal citation, and DOI. wavelength of the radiation emitted by electrons [4]. In 1953, he discussed the design of a practical undulator and experimentally demonstrated the generation of millimeter waves from $3 \mathrm{MeV}$ electron beam [5]. In 1971, Madey realized that the classical formalism of coherent undulator radiation presented by Motz does not account for the stimulated emission processes that can take place due to the interaction between the radiation field and the electron beam. He then proposed the idea of free electron lasers (FEL) based on stimulated emission of bremsstrahlung in undulators [6] and successfully demonstrated the operation of the proposed FEL in 1977 [7].

Gevorgian et al. used the spectrum of the coherent shortwave radiation pulses to obtain the distribution of electrons in an electron bunch and showed that finite length electron bunches would increase the FEL process efficiency [8]. In the same year, Michel pointed out that intense coherent synchrotron radiation from "superbunched" electron beam could be obtained from storage rings also [9]. Coherent synchrotron radiation from short bunches was later observed for the first time by Nakazato and his group at Tohoku University, Japan in 1989 [10]. The theoretical formulation of the multiparticle coherent enhancement of the synchrotron radiation was laid down by Hirschmugl [11]. She showed that the extent of enhancement of spontaneous synchrotron radiation depends on the form-factor of the electron bunch; which gives a measure of how tightly the particles in the bunch are packed around its center of mass as compared to the wavelength of the emitted radiation. She derived the equation for radiated power as: 


$$
\frac{d^{2} I}{d \omega d \Omega}=[N+N(N-1) f(\omega)] P(\omega)
$$

where $\omega$ is the radiation frequency, $P(\omega)$ is the power radiated by a single electron, $N$ is the total number of electrons in the bunch and $f(\omega)$ is its form factor. The form factor of an electron bunch is defined as [11]:

$$
f(\omega)=\frac{1}{N(N-1)} \sum_{j, k=1(j \neq k)}^{N} \exp \left(i \omega\left(r_{j}-r_{k}\right) / c\right)
$$

where $r_{i}$ represents the position of $i$ th electron with respect to the center of mass of the bunch. A more generalized approach to derive the average spectral distribution of coherent radiation, irrespective of the emission process is given in [12]. It is easily deducible from Eq. (1) that $f(\omega)=0$ corresponds to the incoherent limit (the radiation power scales linearly with $N$ ) while $f(\omega)=1$ corresponds to the coherent limit of the emitted radiation (the radiation power scales quadratically with $N$ ). Using this definition of form factor, an electron beam bunch is said to be superradiant if $f(\omega) \rightarrow 1$. Generally, this condition is satisfied if the electron beam bunch is much smaller than the radiation to be generated. However, Gevorgyan et al. showed that the transverse distribution of the electron beam bunch also have a significant effect on the coherence of the emitted radiation [13]. The radiation intensity can be further enhanced by injecting a train of superradiant microbunches (separated by exactly one radiation wavelength) into the undulator. The form factor for a train of microbunches and the process of radiation enhancement has been explained elsewhere [14].

Although the above formulation allows us to understand the enhancement of a particular radiation mode $(\omega)$ by accounting for the phase-space volume occupied by the electrons in the bunch; but the picture of the radiation emission process is incomplete without the time-domain theory of the same. In 1994, Gover et al. provided a quantitative theoretical description of the temporal behavior of the coherent radiation wave-packet emitted by an electron bunch inside a waveguide free electron laser [15]. The time-domain theory to explain the self-amplified spontaneous emission (SASE) process inside a helical undulator and to find the temporal and angular characteristics of the emitted radiation is given in [16]. In this article, we present a qualitative description of the enhancement of undulator radiation due to strong correlation between the radiators, i.e., electrons in a bunch while neglecting the interaction between the radiation field and the particles. The presented method may be used to study the influence of electron beam phase space $\left(x, y, z, x^{\prime}, y^{\prime}, z^{\prime}\right)$ on the trajectory as well as the coherence of the emitted radiation in the time domain. It is based on calculating the waveform (temporal profile) of the emitted radiation as a superposition of waves (with different amplitudes and phases) emitted by different electrons in the bunch. The phase of the electro-magnetic wave arriving at the observer at time $t$ due to the $i$ th particle is calculated with respect to the phase of the wave emitted by an electron moving along the center of mass of the bunch. This phase-difference is because of the relative separation between the electron moving at the center of mass of the bunch and at random position within the bunch.

The formulation to compute the temporal profile of the coherent undulator radiation has been presented in the paper in following manner. In Sec. II, the trajectory of a single electron has been calculated in presence of the hyperbolic fields of the undulator to incorporate the effect of betatron motion. In Sec. III, the Liénard-Wiechert fields have been solved using equation of motion obtained in Sec. II in retarded time to obtain the analytical expression of the emitted radiation. It is shown that the betatron motion of the electron would result in emission of additional higher frequency components. In Sec. IV, the analytical expressions obtained for a single electron has been used to extend the formulation to multiparticle systems. As a case study, the dependency of the intensity of the emitted electromagnetic radiation on the electron beam bunch length has been studied.

\section{EQUATION OF MOTION}

We consider a planar undulator having period $\lambda_{u}$ and peak field along the axis of the undulator as $B_{0}$. The field in the region between the arrays of the undulator magnet can be defined using the scalar potential

$$
V=\frac{B_{0}}{k_{u}} \sinh \left(k_{u} y\right) \sin \left(k_{u} z\right)
$$

where $k_{u}=2 \pi / \lambda_{u}$ is the undulator wave number and $K=0.934 B_{0} \lambda_{u}$ is called as undulator parameter [17]. The magnetic fields along the three axes of the undulator are given by

$$
\begin{aligned}
& B_{x}=0 \\
& B_{y}=-B_{0} \cosh \left(k_{u} y\right) \sin \left(k_{u} z\right) \\
& B_{z}=-B_{0} \sinh \left(k_{u} y\right) \cos \left(k_{u} z\right) .
\end{aligned}
$$

The coupled equation of motion of an electron (energy $\gamma$ and speed $\beta c$ ) having initial phase-space coordinates $\left(x_{0}, y_{0}, 0, v_{x}^{0}, v_{y}^{0}, \beta c\right)$ injected into the undulator are found using the Lorentz force formula:

$$
\dot{\mathbf{v}}=-\frac{e}{\gamma m_{e}}(\mathbf{v} \times \mathbf{B})
$$

From Eq. (5), we get three coupled equations: 


$$
\begin{aligned}
\ddot{x} & =\frac{e B_{0}}{\gamma m_{e}}\left(v_{y} \sinh \left(k_{u} y\right) \cos \left(k_{u} z\right)-v_{z} \cosh \left(k_{u} y\right) \sin \left(k_{u} z\right)\right) \\
\ddot{y} & =-\frac{e B_{0}}{\gamma m_{e}} v_{x} \sinh \left(k_{u} y\right) \cos \left(k_{u} z\right) \\
\ddot{z} & =\frac{e B_{0}}{\gamma m_{e}} v_{x} \cosh \left(k_{u} y\right) \sin \left(k_{u} z\right) .
\end{aligned}
$$

The equation of motion in $x$ can be simplified by defining

$$
\psi=B_{0} \cos \left(k_{u} z\right) \cosh \left(k_{u} y\right)
$$

which results in

$$
\ddot{x}=\frac{e}{\gamma m_{e}} \frac{1}{k_{u}} \frac{d \psi}{d t} .
$$

Integrating Eq. (8) we get:

$$
v_{x}=\frac{e B_{0}}{\gamma m_{e}} \frac{1}{k_{u}} \cos \left(k_{u} z\right) \cosh \left(k_{u} y\right)+\text { constant. }
$$

Using the initial conditions in Eq. (8) we can find the constant term as constant $=v_{x}^{0}-\frac{e B_{0}}{\gamma m_{e}} \frac{1}{k_{u}} \cosh \left(k_{u} y_{0}\right)$. Upon integration of Eq. (9), it can be seen that the effect of the constant term will be to cause a linear drift of the electron away or towards from the axis depending on the slope of the constant term. However; this effect is fully compensated for electron injected at $(x=0, y=0, z=0)$ and partially compensated at the undulator's entrance and exit for particles injected off-axis by steering the electron beam through a combination of dipoles having strength $\frac{1 \text { th }}{4}, \frac{3 \text { th }}{4}$ of the peak magnetic field. We assume that this effect is fully compensated for all the particles irrespective of their initial coordinates.

To proceed further, we obtain the first order solution of electron motion in z-axis by observing that the electron is moving at ultrarelativistic speed along the z-axis and the magnetic field of the undulator deflects the electron along $\mathrm{x}$-axis with a speed $v_{x} \ll \beta c$. Therefore, the velocity and the trajectory of electron in longitudinal direction can be considered to be $v_{z} \equiv \beta c$ and $z=\beta c t$ respectively. It must be noted that $z=\beta c t$ is a valid approximation only if the considered undulator is a weak undulator, i.e., $\frac{K}{\gamma} \rightarrow 0$ or the electron beam is ultrarelativistic, so that any higher order terms may be neglected. We also assume that the undulator period $\lambda_{u}$ is much longer than the beam size in $y$, i.e., $y / \lambda_{u} \rightarrow 0$, which allows us to make two approximations near $y=0$ :

$$
\cosh \left(k_{u} y\right) \rightarrow 1 \text { and } \sinh \left(k_{u} y\right) \rightarrow k_{u} y .
$$

These two approximations reduces the transverse electron motion to following set of two equations:

$$
\begin{gathered}
\dot{x}=\frac{e B_{0}}{\gamma m_{e}} \frac{1}{k_{u}} \cos \left(k_{u} \beta c t\right) \\
\ddot{y}=-\frac{1}{2}\left(\frac{e B_{0}}{\gamma m_{e}}\right)^{2}\left(1+\cos \left(2 k_{u} \beta c t\right)\right) y .
\end{gathered}
$$

Integrating Eq. (10) and sustituting intial values, we get the equation of motion along $\mathrm{x}$-axis:

$$
x-x_{0}=\frac{K}{\gamma \beta k_{u}} \sin \left(k_{u} \beta c t\right)
$$

where $K=\frac{e B_{0}}{m_{e} c k_{u}}$ is called the undulator parameter. The equation of motion along the y-axis described by Eq. (11) belongs to a class of second-order differential equations known as Mathieu differential equation [18]. The canonical form of the Mathieu's differential equation is

$$
\frac{d^{2} y}{d \tau^{2}}=-(a-2 q \cos (2 \tau)) y .
$$

The solution of Eq. (13) is expressed in terms of linear combination of Mathieu odd and even functions. Odd Mathieu functions represented by $S(a, q, z)$ are also known as Mathieu sine function with characteristic value $a$ and parameter $q$ while Even Mathieu functions represented by $C(a, q, z)$ are also called Mathieu cosine functions. Upon substituting $\tau=k_{u} \beta c t$, Eq. (11) becomes:

$$
\frac{d^{2} y}{d \tau^{2}}=-\frac{1}{2}\left(\frac{K}{\gamma \beta}\right)^{2}(1+\cos (2 \tau)) y .
$$

Equation (14) is a special case of Eq. (13) with

$$
\begin{aligned}
& a=\frac{1}{2}\left(\frac{K}{\gamma \beta}\right)^{2} \\
& q=-0.5 a .
\end{aligned}
$$

Therefore, the solution of Eq. (11) can be represented as a linear combination of Mathieu functions as:

$y(t)=c_{1} C\left(a,-\frac{a}{2}, k_{u} \beta c t\right)+c_{2} S\left(a,-\frac{a}{2}, k_{u} \beta c t\right)$

and the velocity is given by its derivative as:

$\dot{y}(t)=k_{u} \beta c\left(c_{1} C^{\prime}\left(a,-\frac{a}{2}, k_{u} \beta c t\right)+c_{2} S^{\prime}\left(a, \frac{a}{2}, k_{u} \beta c t\right)\right)$

where $C^{\prime}\left(a,-a / 2, k_{u} \beta c t\right)$ and $S^{\prime}\left(a,-a / 2, k_{u} \beta c t\right)$ are the derivatives of the Mathieu functions. The coefficients $c_{1}$ and $c_{2}$ can be obtained by applying the initial conditions to Eq. (15) and (16). The coefficients are then given by: 


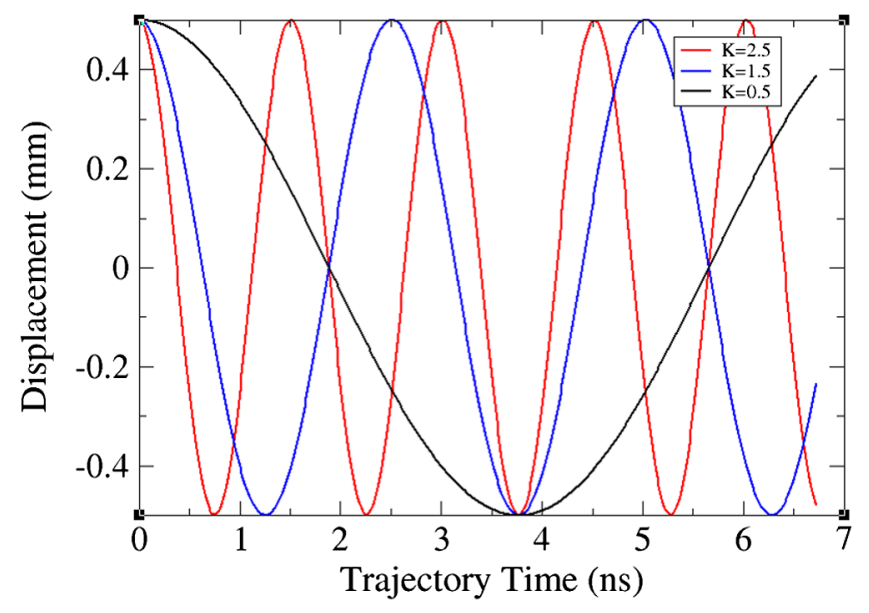

FIG. 1. Betatron motion of an electron $(E=8 \mathrm{MeV})$ injected $0.5 \mathrm{~mm}$ off-axis for different $\mathrm{K}$.

$$
\begin{aligned}
c_{1} & =\frac{y_{0}}{C(a,-0.5 a, 0)} \\
c_{2} & =\frac{v_{y}^{0}}{k_{u} \beta c S^{\prime}(a,-0.5 a, 0)}
\end{aligned}
$$

The benefit of writing the equation of motion along $y$-axis in the form of Eq. (15) is that the betatron motion of the electron can be easily accounted for as a function of $z$ or $t$ without averaging over the length of the undulator. As an example, we consider an electron injected $0.5 \mathrm{~mm}$ off-axis in $\mathrm{y}$ and look at its motion over the length of an undulator with $N_{u}=42, \lambda_{u}=0.048 \mathrm{~mm}$ and variable undulator parameter $K$ (Fig. 1). As it is expected, the focusing strength of the undulator and the number of oscillations of the electron increases with undulator parameter $K$ [19]. The region in which the obtained solutions are valid (region where linear approximation agrees well with hyperbolic field) is shown in Fig. 2. Note that a similar expression for the trajectory has been mentioned in [20].

It is important to note that the first order solution for motion along z-axis is applicable strictly for weak

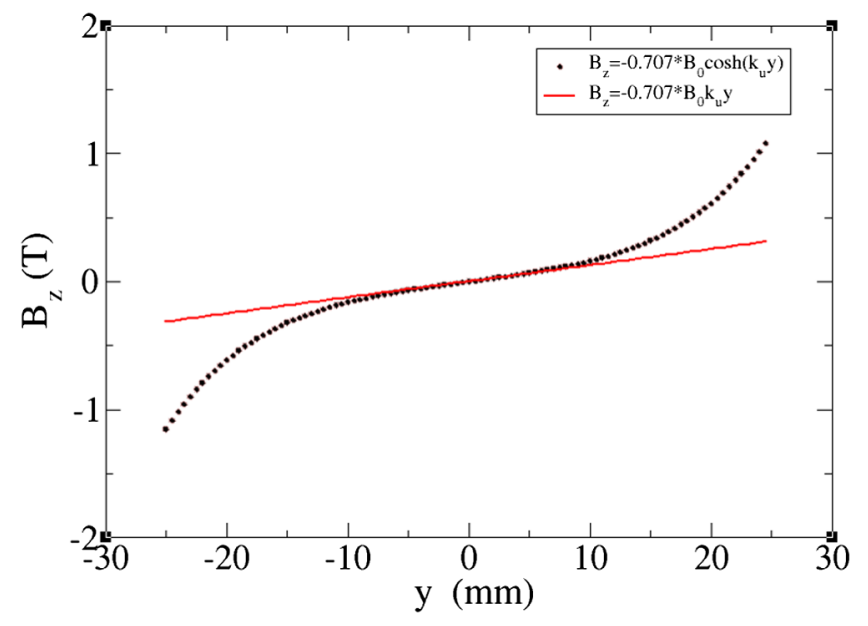

FIG. 2. Variation of magnetic field along y near the region of approximation $k_{u} y \rightarrow 0 B_{z} \rightarrow k_{u} y \cos \left(k_{u} z\right)$.

undulators i.e., $K / \gamma \rightarrow 0$ or for ultrarelativistic particles and a more general solution is given by following set of equations [17]:

$$
\begin{gathered}
z(t)=\tilde{v}_{z} t-\frac{K^{2}}{8 \gamma^{2} k_{u}} \sin \left(2 \omega_{u} t\right) \\
v_{z}(t)=\left(1-\frac{1}{2 \gamma^{2}}\left(1+\frac{K^{2}}{2}\right)\right) c-\frac{c K^{2}}{4 \gamma^{2}} \cos \left(2 \omega_{u} t\right)
\end{gathered}
$$

where $\omega_{u}=k_{u} \tilde{\beta} c$ is the undulator angular frequency and $\tilde{\beta}$ is the average longitudinal velocity given by:

$$
\tilde{\beta}=\left(1-\frac{1}{2 \gamma^{2}}\left(1+\frac{K^{2}}{2}\right)\right)
$$

Therefore, the motion of an electron moving inside an undulator is defined by following equations:

$$
\begin{aligned}
\mathbf{r}_{\mathbf{s}}(t) & =\left(x_{0}+\frac{K}{\gamma \beta k_{u}} \sin \left(k_{u} \beta c t\right), c_{1} C\left(a,-\frac{a}{2}, k_{u} \beta c t\right)+c_{2} S\left(a,-\frac{a}{2}, k_{u} \beta c t\right),\left(1-\frac{1}{2 \gamma^{2}}\left(1+\frac{K^{2}}{2}\right)\right) c t-\frac{K^{2}}{8 \gamma^{2} k_{u}} \sin \left(2 \omega_{u} t\right)\right) \\
\boldsymbol{\beta}(t) & =\left(\frac{K}{\gamma} \cos \left(k_{u} \beta c t\right) \cosh \left(k_{u} y\right), k_{u} \beta c_{1} C^{\prime}\left(a,-\frac{a}{2}, k_{u} \beta c t\right)+k_{u} \beta c_{2} S^{\prime}\left(a, \frac{a}{2}, k_{u} \beta c t\right), 1-\frac{1}{2 \gamma^{2}}\left(1+\frac{K^{2}}{2}\right)-\frac{K^{2} \beta}{4 \gamma^{2}} \cos \left(2 \omega_{u} t\right)\right) \\
\dot{\boldsymbol{\beta}}(t) & =\frac{e B_{0}}{\gamma m_{e}}\left(\beta_{y} \sinh \left(k_{u} y\right) \cos \left(k_{u} \beta c t\right)-\beta \cosh \left(k_{u} y\right) \sin \left(k_{u} \beta c t\right),-\frac{1}{2} \frac{K k_{u}}{\gamma}\left(1+\cos \left(2 k_{u} \beta c t\right)\right) y, \beta_{x} \cosh \left(k_{u} y\right) \sin \left(k_{u} \beta c t\right)\right) .
\end{aligned}
$$

\section{UNDULATOR RADIATION FROM SINGLE PARTICLE}

In the literature, the temporal profile of the electric field of the emitted undulator radiation is obtained for electron moving close to undulator axis $(y \rightarrow 0)$ [21]. In the previous section, the equation of motion of an electron injected with arbitrary initial coordinates was derived in a different way enabling us to include the effect of betatron motion. In this section, we utilize the new set of equation of 
motion to solve Lienard-Wiechert Fields in retarded time $\left(t_{r}\right)$ and relate $t_{r}$ to the observer time to find the temporal profile of the detected wave. The radiation part of the Lienard-Wiechert fields is given as [22]:

$$
\mathbf{E}(\mathbf{r}, \mathbf{t})=\frac{q}{4 \pi \epsilon_{0} c}\left(\frac{\hat{\mathbf{n}} \times((\hat{\mathbf{n}}-\boldsymbol{\beta}) \times \dot{\boldsymbol{\beta}})}{|\mathbf{R}|(1-\hat{\mathbf{n}} \cdot \boldsymbol{\beta})^{3}}\right)_{t_{r}}
$$

where $\mathbf{R}=\mathbf{r}-\mathbf{r}_{\mathrm{s}}$ is the separation between the observer (r) and the electron $\left(\mathbf{r}_{\mathbf{s}}\right)$ and $\hat{\mathbf{n}}=\frac{\mathbf{R}}{R}$ is the normal vector pointing from the electron to the observer. $\boldsymbol{\beta}$ and $\dot{\boldsymbol{\beta}}$ are the normalized velocity and acceleration of the electron at time $t_{r}$.

If we assume that the observer is placed extremely far from the region where the electron performed its wiggling motion, i.e., $r \gg r_{s}$, then $\mathbf{R} \simeq \mathbf{r}$ and $\hat{\mathbf{n}}=\hat{\mathbf{r}}$. The radiation emitted by the electron at position $\mathbf{r}_{\mathbf{s}}\left(t_{r}\right)$ is detected by an observer placed at $\mathbf{R}$ at time $t$, whose coordinates in spherical system are given by:

$$
\begin{aligned}
\mathbf{R} & =r(\sin \theta \cos \phi, \sin \theta \sin \phi, \cos \theta) \\
\hat{\mathbf{n}} & =(\sin \theta \cos \phi, \sin \theta \sin \phi, \cos \theta) .
\end{aligned}
$$

Using Eq. (II) and (22), the solution of Eq. (21) is given as the following:

$$
\mathbf{E}(\mathbf{r}, \mathbf{t})=\frac{-e}{4 \pi \epsilon_{0} c r}\left[\frac{1}{(1-\hat{\mathbf{n}} \cdot \boldsymbol{\beta})^{3}}\left(E_{x}, E_{y}, E_{z}\right)\right]_{t_{r}}
$$

where

$$
1-\hat{\mathbf{n}} \cdot \boldsymbol{\beta}=1-\beta \cos \theta-\beta_{x} \cos \phi \sin \theta-\beta_{y} \sin \phi \sin \theta
$$

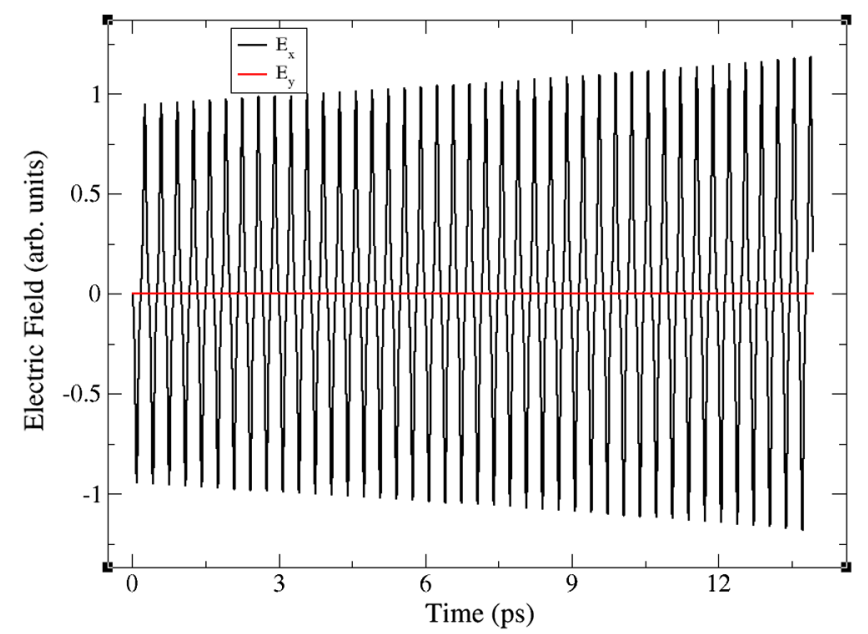

$$
\begin{aligned}
E_{x}= & \dot{\beta}_{x} \cos ^{2} \theta+\cos \theta\left(\dot{\beta}_{z} \beta_{x}-\dot{\beta}_{x} \beta-\dot{\beta}_{z} \cos \phi \sin \theta\right) \\
+ & \sin \phi \sin \theta\left(\dot{\beta}_{y} \beta_{x}-\dot{\beta}_{x} \beta_{y}-\dot{\beta}_{y} \cos \phi \sin \theta\right. \\
+ & \left.\dot{\beta}_{x} \sin \theta \sin \phi\right) \\
E_{y}= & \dot{\beta}_{y} \cos ^{2} \theta+\cos \phi \sin \theta\left(-\dot{\beta}_{y} \beta_{x}+\dot{\beta}_{x} \beta_{y}\right. \\
& \left.\quad+\dot{\beta}_{y} \cos \phi \sin \theta-\dot{\beta}_{x} \sin \phi \sin \theta\right) \\
& +\cos \theta\left(\dot{\beta}_{z} \beta_{y}-\dot{\beta}_{y} \beta-\dot{\beta}_{z} \sin \phi \sin \theta\right) \\
E_{z}= & \sin \theta\left(\cos \phi\left(-\dot{\beta}_{z} \beta_{x}+\dot{\beta}_{x} \beta-\dot{\beta}_{x} \cos \theta\right)\right. \\
+ & \left.\left(-\dot{\beta}_{z} \beta_{y}+\dot{\beta}_{y} \beta-\dot{\beta}_{y} \cos \theta \sin \phi+\dot{\beta}_{z} \sin \theta\right)\right) .
\end{aligned}
$$

It is important to note that Eq. (23) corresponds to solution in the retarded time. The retarded time and the observer time are related by a simple equation:

$$
t=t_{r}+\frac{\left|\mathbf{r}-\mathbf{r}_{\mathbf{s}}\left(\mathbf{t}_{\mathbf{r}}\right)\right|}{c}
$$

As an application of the theory presented in this section, we discuss two cases. In the first case, we choose $x_{0}=0, y_{0}=0, v_{y}^{0}=0, \theta=0$, and $\phi=0$, and show that Eq. (23) reduces to the case when observer is located along the axis of the undulator:

$$
\mathbf{E}(\mathbf{r}, t)=\frac{-e}{4 \pi \epsilon_{0} c r}\left(\frac{\dot{\beta}_{x}}{(1-\beta)^{2}}+\frac{\dot{\beta}_{z} \beta_{x}}{(1-\beta)^{3}}, 0,0\right)_{t_{r}} .
$$

In the second case, we choose $x_{0}=0, y_{0} \neq 0, v_{y}^{0}=0, \theta=0, \phi=0$ and show how the emitted undulator radiation is affected due to betatron oscillations. In this case, Eq. (23) becomes following:

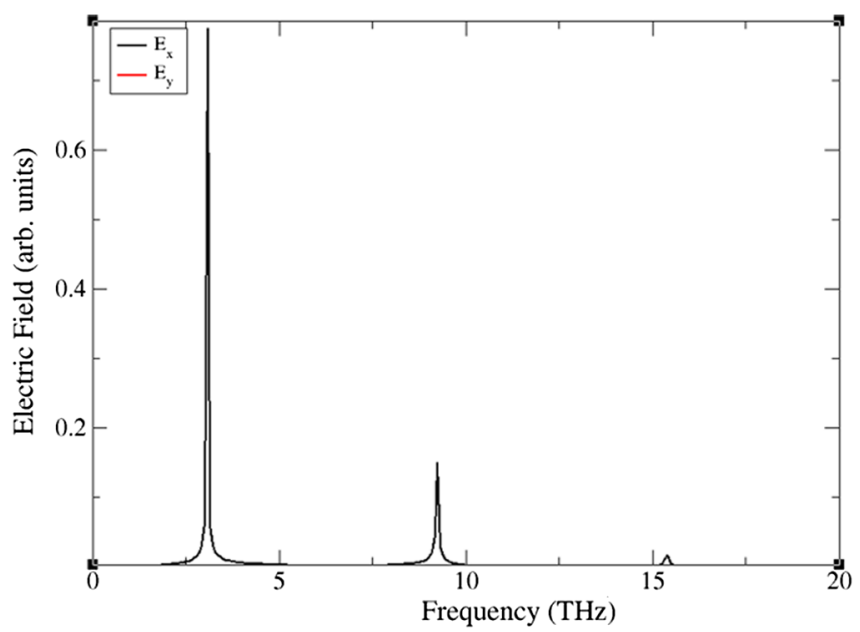

FIG. 3. Temporal profile and spectrum of radiation emitted by an electron injected on-axis of an undulator with $K=0.5$. 

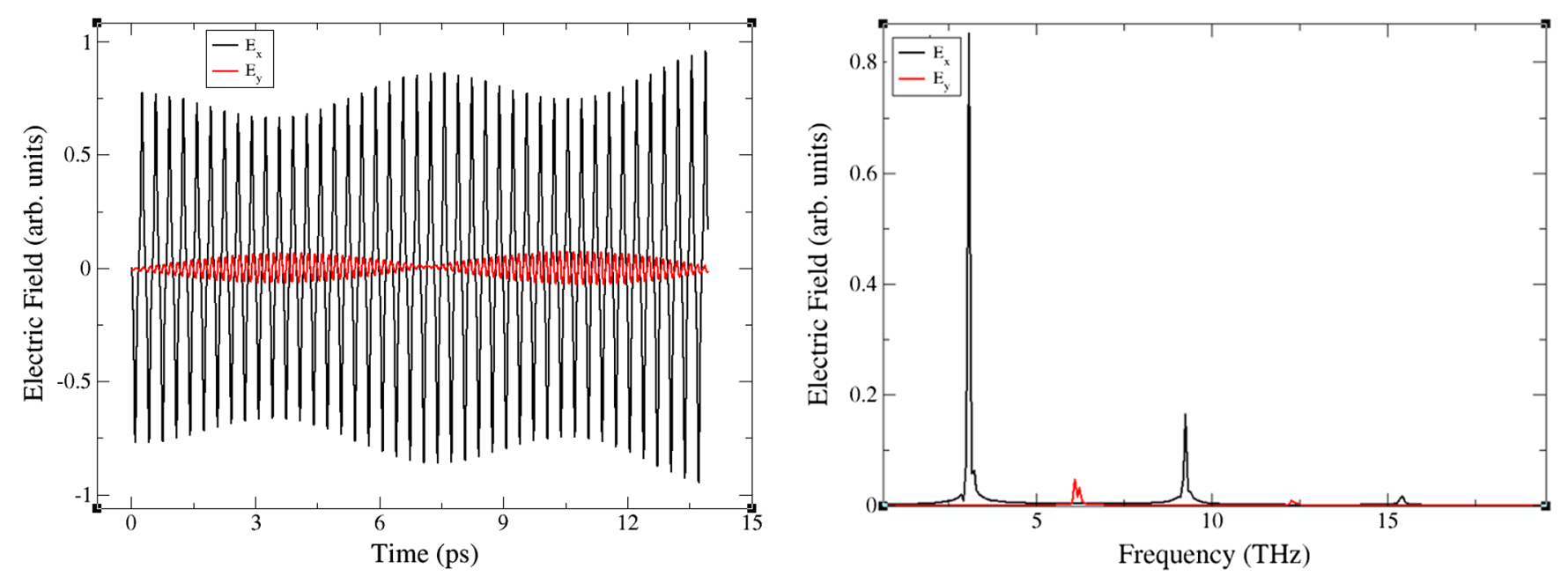

FIG. 4. Temporal profile and spectrum of radiation emitted by an electron injected off-axis of an undulator with $K=0.5$.
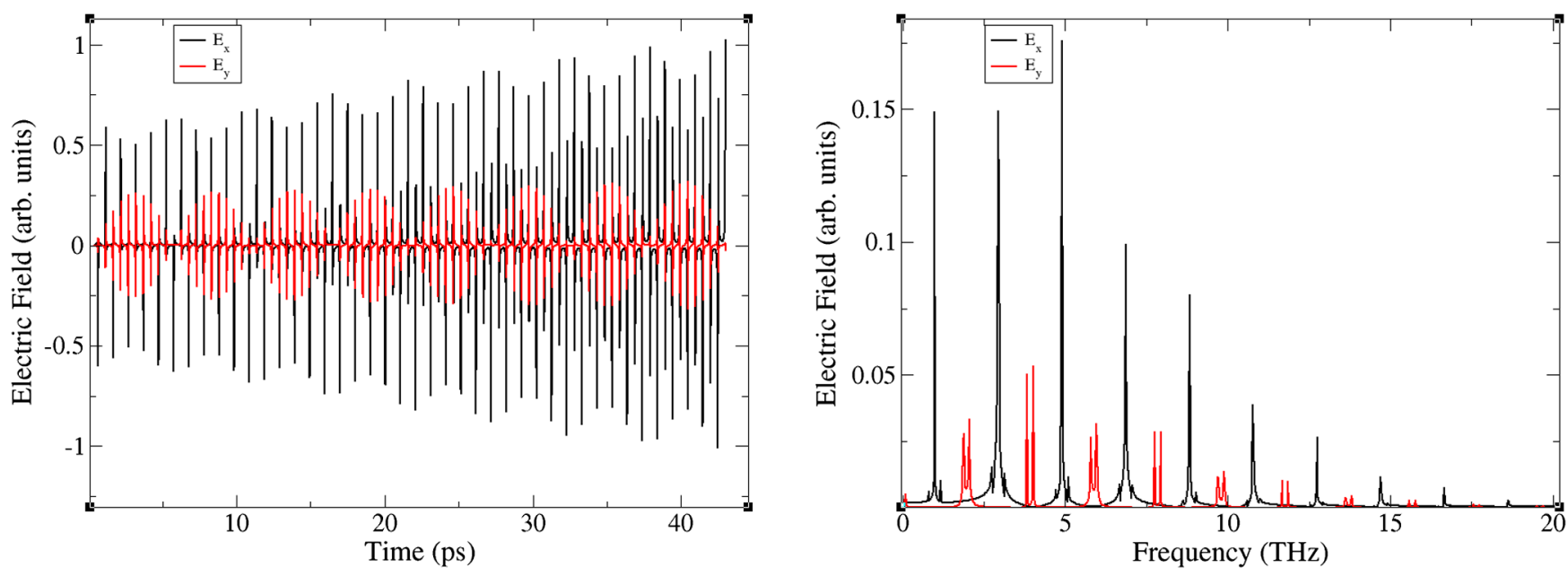

FIG. 5. Temporal profile and spectrum of radiation emitted by an electron injected off-axis in an undulator with $K=2.5$.

$$
\begin{aligned}
\mathbf{E}(\mathbf{r}, t)= & \frac{-e}{4 \pi \epsilon_{0} c r}\left(\frac{\dot{\beta}_{x}}{(1-\beta)^{2}}+\frac{\dot{\beta}_{z} \beta_{x}}{(1-\beta)^{3}},\right. \\
& \left.\frac{\dot{\beta}_{y}}{(1-\beta)^{2}}+\frac{\dot{\beta}_{z} \beta_{y}}{(1-\beta)^{3}}, 0\right)_{t_{r}}
\end{aligned}
$$

Equation (24), Eq. (25), and Eq. (26) have been analyzed using the Wolfram Alpha [23] mathematical tool to find the variation of electric field with time and its spectrum for $8 \mathrm{MeV}$ electron injected into a 42 period undulator with period length $48 \mathrm{~mm}$ and $K=0.5$. Figures 3 and 4 show the results obtained for radiation emitted by electron injected on-axis and off-axis respectively. It is observed that the electron injected along the axis of the undulator has only x-component; however it is not true for the electron injected off-axis. The radiation emitted by second electron has both $E_{x}$ and $E_{y}$ components and the amplitude of the radiation fluctuates due to betatron oscillations performed by the electron.
It is interesting to see the effect of increasing the undulator field strength. As $K$ is increased, the betatron motion becomes more pronounced, resulting in increase in the amplitude of the $E_{y}$ component (Fig. 5). The effect of the large value of undulator parameter is that the spectrum is populated with higher harmonics of the fundamental [17] and the effect of injecting electron off-axis $(y \neq 0)$ in an undulator with large $K$ is that the frequencies corresponding to the betatron motion are also observed.

\section{MULTIPARTICLE ANALYSIS}

In the last two sections, preliminary calculations required to extend the single particle time domain theory for the case of many particles present inside an electron bunch has been presented. Generalized set of equations were required so as to include the effect of hyperbolic field profiles on the particle trajectory as well as the emitted radiation. The formulation to extend the analysis to multiparticle systems 
is set in the following manner (schematic shown in Fig. 6). Let us consider a monoenergetic electron bunch consisting of $N_{e}$ electrons. The radiation emitted by different electrons at the retarded time $t_{r}$ will reach the observer at different times because of the finite size of the electron bunch. Therefore, the emitted radiation is detected by an observer placed at $\mathbf{r}$ in a time duration $t \pm \delta t$. Let us assume that the radiation emitted by $i$ th particle and by the particle at the center of mass of the bunch at retarded time $t_{r}$ reach the observer at time $t-\delta t_{i}$ and $t$ respectively. If at the time of emission, the spatial coordinate of the $i$ th electron is represented by $\mathbf{r}_{\mathbf{i}}\left(\mathbf{t}_{\mathbf{r}}\right)$ and the spatial coordinate of the center of mass is represented by $\mathbf{r}_{\mathbf{c o m}}\left(\mathbf{t}_{\mathbf{r}}\right)$, then the total path length traveled by the two waves is given by:

$$
\begin{gathered}
c\left(t-\delta t_{i}-t_{r}\right)=\left|\mathbf{r}-\mathbf{r}_{\mathbf{i}}\left(t_{r}\right)\right| \\
c\left(t-t_{r}\right)=\left|\mathbf{r}-\mathbf{r}_{\text {com }}\left(t_{r}\right)\right| .
\end{gathered}
$$

The path-difference between the radiation emitted by the $i$ th particle and the particle at center of mass is given by the difference between Eq. (28) and Eq. (27).

$$
c \delta t_{i}=\left|\mathbf{r}-\mathbf{r}_{\mathbf{c o m}}\left(t_{r}\right)\right|-\left|\mathbf{r}-\mathbf{r}_{\mathbf{i}}\left(t_{r}\right)\right|
$$

Let us assume that the observer is situated very far from the source of the radiation, i.e., the bunch of electrons $\left(|\mathbf{r}| \gg\left|\mathbf{r}_{\mathbf{i}}\right|\right.$ and $\left.|\mathbf{r}| \gg\left|\mathbf{r}_{\text {com }}\right|\right)$. Under this approximation, we can assume that $\frac{\left|\mathbf{r}_{\mathbf{i}}\right|}{|\mathbf{r}|} \rightarrow 0$ and $\frac{\left|\mathbf{r}_{\text {com }}\right|}{|\mathbf{r}|} \rightarrow 0$. Therefore,

$$
\begin{aligned}
\left|\mathbf{r}-\mathbf{r}_{\mathbf{i}}\right| & =\sqrt{\mathbf{r} \cdot \mathbf{r}-2 \mathbf{r} \cdot \mathbf{r}_{\mathbf{i}}+\mathbf{r}_{\mathbf{i}} \cdot \mathbf{r}_{\mathbf{i}}} \\
& =\mathbf{r} \sqrt{1-2 \frac{\hat{\mathbf{n}} \cdot \mathbf{r}_{\mathbf{i}}}{r}+\frac{\mathbf{r}_{\mathbf{i}} \cdot \mathbf{r}_{\mathbf{i}}}{r^{2}}} \\
& \approx r\left(1-\frac{\hat{\mathbf{n}} \cdot \mathbf{r}_{\mathbf{i}}}{r}+\frac{r_{i}^{2}}{2 r^{2}}\right) .
\end{aligned}
$$

Similarly,

$$
\left|\mathbf{r}-\mathbf{r}_{\mathbf{c o m}}\right| \approx r\left(1-\frac{\hat{\mathbf{n}} \cdot \mathbf{r}_{\mathbf{c o m}}}{r}+\frac{r_{\mathrm{com}}^{2}}{2 r^{2}}\right) .
$$

Using the result obtained in Eq. (30) and Eq. (31) in Eq. (29). we get:

$$
c \delta t_{i}=\hat{\mathbf{n}} \cdot\left(\mathbf{r}_{\mathbf{i}}-\mathbf{r}_{\mathrm{com}}\right)+\frac{1}{2 r}\left(r_{\mathrm{com}}^{2}-r_{i}^{2}\right) .
$$

The following remarks can be made about the two terms appearing in Eq. (32):

(1) $\hat{\mathbf{n}} \cdot\left(\mathbf{r}_{\mathbf{i}}-\mathbf{r}_{\text {com }}\right)$ : The path difference between the radiation wave packets emitted by any two electrons of a particular bunch depends on the relative separation between the electrons (which may vary as the electron bunch traverse through the insertion device) along the direction of the observer. This means that if the observer is located along the axis of the insertion device, the path-difference because of this term would be solely dependent on the longitudinal separation between the electrons. Therefore, the on-axis radiation is primarily affected by the longitudinal distribution of the electron bunch only. However, if we move off-axis, the transverse beam distribution of the electron bunch starts becoming important.

(2) $\frac{1}{2 r}\left(r_{c o m}^{2}-r_{i}^{2}\right)$ : This term brings out the fact that the size of the electron beam becomes irrelevant if the observer is placed extremely far from the entire trajectory of the electron beam. However, as the observer moves closer to the electron beam, the emitted waves can no longer be considered as the plane wave and path-difference due to curvature of the spherical waves starts becoming important. It must also be noted that if only the on-axis radiation is considered, then contribution to path-difference from transverse beam distribution is negligible. Thus, the effect of transverse beam distribution on the emitted radiation is a second order effect and decays rapidly with the observer distance.

Equation (32) can also be used to find the phasedifference between the waves detected by an observer if the wavelength of the emitted wave is known. The wavelength $\lambda_{n}$ of the $n$th harmonic of the radiation emitted by a wiggling electron is given by [17]:

$$
\lambda_{n}=\frac{\lambda_{u}}{2 n \gamma^{2}}\left(1.0+\frac{K^{2}}{2}+\gamma^{2} \theta^{2}\right)
$$

The phase-difference is then given by:

$$
\delta \Phi_{i}=2 \pi c \frac{\delta t_{i}}{\lambda_{n}} .
$$

The temporal profile of the undulator radiation emitted by the bunch of electrons is then simply given by the superposition of waves emitted by individual electrons in the bunch:

$$
\mathbf{E}(r, t)=\sum_{i=1}^{N_{e}} \mathbf{E}_{\mathbf{i}}\left(r, t+\delta t_{i}\right)
$$

where $\mathbf{E}_{\mathbf{i}}(r, t)$ is found using Eq. (23) and Eq. (24). Let us now consider the case of an ultrarelativistic $(\gamma \gg 1)$ electron bunch wiggling inside a planar weak undulator $(K \approx 1)$. For such a system, the maximum deflection of the electron beam from its axis is extremely small, i.e., $\frac{K}{k_{u} \gamma} \rightarrow 0$ [Eq. (II)] and therefore higher order terms can be neglected. The trajectory of the particle at the center of mass of the electron bunch inside the undulator is given by [17]: 


$$
\mathbf{r}_{\mathbf{c o m}}\left(t_{r}\right)=\left[\frac{K}{\gamma \beta k_{u}} \sin \left(\omega_{u} t_{r}\right), 0, \tilde{\beta} c t_{r}\right]
$$

If the initial coordinates of the $i$ th particle with respect to center of mass were $\left(\delta x_{i}, \delta y_{i}, \delta z_{i}\right)$, then its trajectory is given by $\mathbf{r}_{\mathbf{i}}\left(t_{r}+\delta z_{i} / \beta c\right)$. We use Eq. (36) and Eq. (20) in Eq. (32) to find the equation for path-difference between the radiation emitted by particle located at the center of mass of the bunch and $i$ th particle. If $\mathbf{R}=r[\sin \theta \cos \phi, \sin \theta \sin \phi, \cos \theta]$, then the first term of Eq. (32) can be written as:

$$
\begin{aligned}
1 . \hat{\mathbf{n}} \cdot\left(\mathbf{r}_{\mathbf{i}}-\mathbf{r}_{\mathbf{c o m}}\right)= & \delta z_{i} \cos \theta+\sin \phi \sin \theta\left(c_{1} C\left(a,-\frac{a}{2}, k_{u} \beta c\left(t+\frac{\delta z_{i}}{\beta c}\right)\right)+c_{2} S\left(a,-\frac{a}{2}, k_{u} \beta c\left(t+\frac{\delta z_{i}}{\beta c}\right)\right)\right. \\
& +\cos \phi \sin \theta\left(x_{0}+\frac{2 K}{\gamma \beta k_{u}} \cos \left(\omega_{u}\left(\frac{\delta z_{i}}{2 \beta c}+t_{r}\right)\right) \sin \left(\frac{\omega_{u} \delta z_{i}}{2 \beta c}\right)\right)
\end{aligned}
$$

and the second term (ignoring all terms with $K^{2} / \gamma^{2}$ ) as:

$$
\text { 2. } \begin{aligned}
\frac{-1}{2 r}\left(r_{\mathrm{com}}^{2}-r_{i}^{2}\right)= & \frac{1}{2 r}\left\{\left(\delta x_{i}^{2}+\delta z_{i}^{2}\right)+2 \beta c t_{r} \delta z_{i}+\frac{2 K \delta x_{i}}{\gamma \beta k_{u}} \sin \left(\omega_{u}\left(t_{r}+\frac{\delta z_{i}}{\beta c}\right)\right)+\left(c_{1} C\left(a,-\frac{a}{2}, k_{u} \beta c t\right)\right.\right. \\
& \left.\left.+c_{2} S\left(a,-\frac{a}{2}, k_{u} \beta c t\right)\right)^{2}\right\} .
\end{aligned}
$$

The above two equations can be used to evaluate the pathdifference occurring due to different electron beam parameters. Let us consider a very simple case to check the validity of the presented method. It is well known that the radiation emitted along the axis of the undulator slips ahead of the electron by one radiation wavelength per undulator period. Therefore; if we inject two electrons separated by $\lambda_{r}$, then the radiation emitted along the axis of the undulator by trailing electron will constructively interfere with the radiation emitted by the leading electron. It can be shown using Eq. (37) that the phase difference of the radiation emitted by any of the two electrons will have a phase difference of $\pi$ with the radiation emitted by an electron moving along center of mass. Therefore, the phase difference between the radiation emitted by these two electrons will be $2 \pi$. It is interesting to observe that the phase difference between the radiation emitted along the axis of the undulator by an electron in a bunch longitudinally separated from center of mass by $\delta z$ will be equal to the phase difference between the radiation emitted by electrons separated along $\mathrm{X}$-axis by $\sqrt{2 r \delta z}$. This means that transverse beam size can be much larger in $\mathrm{x}$-dimension than the longitudinal beam size; subjected to the condition that the beam size in $\mathrm{x}$-dimension is small enough to avoid the "roll-off" effects. This logic is not applicable along the yaxis because of the presence of hyperbolic fields, which will not allow the electron to follow a stable trajectory through the undulator.

As an example of application to multi-particle systems, let us assume that a bunch of $N_{e}$ electrons having $\gamma m_{0} c^{2}=$ $8.511, \sigma_{x}=0.5 \mathrm{~mm}, \sigma_{y}=0.5 \mathrm{~mm}$ with variable $\sigma_{z}$ is traversing inside an undulator having $K \simeq 0.5, \lambda_{u}=48 \mathrm{~mm}$. It is important to realize that the electron beam is traveling

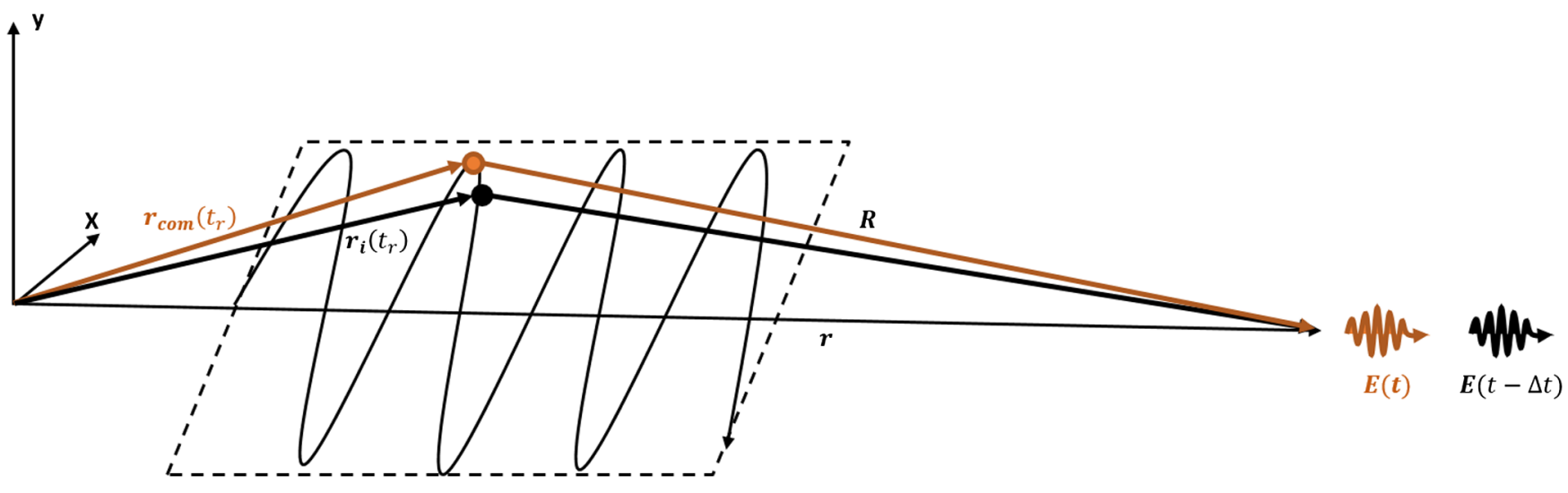

FIG. 6. Radiation emitted by electrons positioned at $\mathbf{r}_{\mathbf{c o m}}\left(\mathbf{t}_{\mathbf{r}}\right)$ and $\mathbf{r}_{\mathbf{i}}\left(\mathbf{t}_{\mathbf{r}}\right)$ reaches the observer situated at $\mathbf{R}$ at $t-\delta t$ and $t$ respectively. The overall profile of the radiation is superposition of waves emitted by all electrons with respect to the center of mass with "time-delay" accounted for as a phase-difference. 

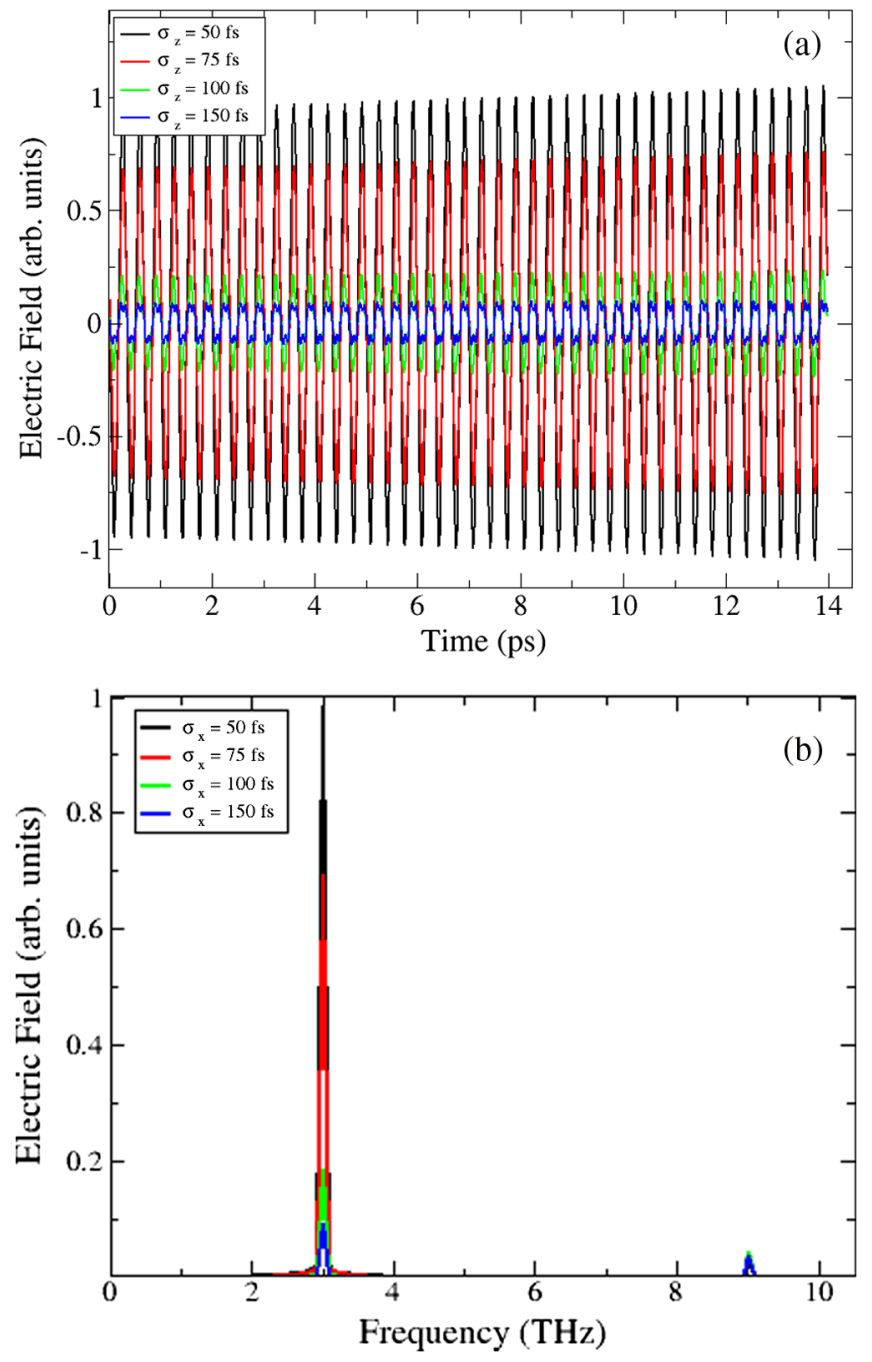

FIG. 7. Variation of temporal profile and spectrum of radiation emitted by an electron beam with bunch length. The values are normalized to the peak field obtained for the case of $\sigma_{z}=50 \mathrm{fs}$ electron bunch.

towards the observer; therefore; the longitudinal distribution is expanded by a combined Lorentz and Doppler factors (to perform the calculation in retarded time) by a factor $2.0 \gamma^{2} /\left(1+K^{2} / 2+\gamma^{2} \theta^{2}\right)$. We consider only the onaxis radiation, i.e., $(\theta=0, \phi=0)$ which is detected by an observer at a distance of $R_{\text {obs }}=10.0 \mathrm{~m}$ from the undulator's entrance. The temporal profile and the spectrum of the radiation emitted for this case is shown in Fig. 7. The results shown in Fig. 7 agrees with the fact that as the beam bunch length is reduced to much below the radiation wavelength $\left(\sigma_{z} \rightarrow 50 \mathrm{fs}\right)$, the emitted radiation approaches the coherent limit and achieves the maximum possible intensity. In Fig. 7(a), the radiation obtained for the case of $\sigma_{z}=50 \mathrm{fs}$ is considered to be fully coherent. Therefore, the amplitudes of the electric field obtained for all the cases have been normalized to this value. Normalization of the field amplitudes help in estimating the bunching factor by directly looking at the Fourier Transform of the temporal profile [shown in Fig. 7(b)]. This means that the form factor of the bunches with bunch length $50 \mathrm{fs}, 75 \mathrm{fs}, 100 \mathrm{fs}, 150 \mathrm{fs}$ has values $1,0.7,0.2$, and 0.1 , respectively.

\section{CONCLUSION}

The extent of the coherence of the radiation emitted by an accelerated electron bunch is usually understood in terms of the form-factor of the electron bunch. The formfactor provides a direct information about the power radiated into a particular radiation mode $\omega$ while accounting for the distribution of the electrons around the center of mass of the bunch. Although the frequency domain approach is much straightforward; a time-domain approach to understand the process of emission of radiation from a bunch provides a deeper insight. The effect of the trajectory followed by an electron inside the undulator is directly observable via the variation of the envelope of the electric field with time. Fourier transform of the temporal profile helps in extracting and understanding the spectrum of the emitted radiation. The accuracy of the obtained results depends on the analytical model used to define the trajectory of the electron. For example, the betatron motion of an electron was expressed in time-domain in terms of the Mathieu functions which allowed in understanding its effects on the radiation temporal profile and the spectrum. We showed that the single particle analysis can be extended to multiparticle systems by correctly accounting for the phase difference between the radiation emitted by different electrons. It was realized that the phase difference is dependent on both, i.e., the distribution of the electrons around the center of mass of the bunch and the angle at which the radiation is detected by an observer. For the case of on-axis radiation, the effect of the transverse beam size is only second-order and can be neglected if the observer is extremely far from the undulator. However, for the case of off-axis radiation, the transverse beam distribution cannot be neglected and the phase-difference arising due to relative separation between the electrons and the center of mass in the transverse plane must be correctly evaluated. Using the presented formulation, we evaluated the temporal profile of the radiation emitted by electron bunches with different longitudinal distribution and obtained their form factors by normalizing the amplitudes with respect to the case of a superradiant electron bunch.

\section{ACKNOWLEDGMENTS}

The authors would like to acknowledge Board of Research in Nuclear Sciences (BRNS), India for providing financial support. The help received from Mr. Rohan Biswas, IUAC for discussions on various aspects of this article is appreciated. 
[1] D. Iwanenko and I. Pomeranchuk, On the maximal energy attainable in a betatron, Phys. Rev. 65, 343 (1944).

[2] J. Schwinger, Phys. Rev. Lett. 75, 1912 (1949).

[3] V. L. Ginzburg, On the radiation of microradiowaves and their absorption in the air, Isvestia Akademii Nauk SSSR 11, 165 (1947).

[4] H. Motz, Applications of the radiation from fast electron beams, J. Appl. Phys. 22, 527 (1951).

[5] H. Motz, W. Thon, and R. N. Whitehurst, Experiments on radiation by fast electron beams, J. Appl. Phys. 24, 826 (1953).

[6] J. M. Madey, Stimulated emission of bremsstrahlung in a periodic magnetic field, J. Appl. Phys. 42, 1906 (1971).

[7] D. A. G. Deacon, L. R. Elias, J. M. J. Madey, G. J. Ramian, H. A. Schwettman, and T. I. Smith, First Operation of a Free-Electron Laser, Phys. Rev. Lett. 38, 892 (1977).

[8] L. A. Gevorgyan and N. K. Zhevago, Coherent radiation of electron bunches in a free electron laser, Dokladi Akad. Nauk SSSR 267, 599 (1982).

[9] F. C. Michel, Intense Coherent Submillimeter Radiation in Electron Storage Rings, Phys. Rev. Lett. 48, 580 (1982).

[10] T. Nakazato, M. Oyamada, N. Niimura, S. Urasawa, O. Konno, A. Kagaya, R. Kato, T. Kamiyama, Y. Torizuka, T. Nanba, Y. Kondo, Y. Shibata, K. Ishi, T. Ohsaka, and M. Ikezawa, Observation of Coherent Synchrotron Radiation, Phys. Rev. Lett. 63, 1245 (1989).

[11] C. J. Hirschmugl, M. Sagurton, and G. P. Williams, Multiparticle coherence calculations for synchrotron-radiation emission, Phys. Rev. A 44, 1316 (1991).

[12] N. A. Korkhmazian, L. A. Gevorgian, and M. L. Petrosian, Influence of probability density of electrons on coherence of the bunch radiation, Zh. Tekh. Fiz. 47, 1583 (1977).
[13] H. Gevorgyan and L. Gevorgian, Coherent radiation characteristics of modulated electron bunch formed in stack of two plates, Nucl. Instrum. Methods Phys. Res., Sect. B 402, 126 (2017).

[14] V. Joshi, J. Karmakar, N. Kumar, B. Karmakar, S. Tripathi, S. Ghosh, R. K. Bhandari, D. Kanjilal, A. Aryshev, and J. Urakawa, Theoretical and simulation study of 'Comb' electron beam and $\mathrm{THz}$ generation, Nucl. Instrum. Methods Phys. Res., Sect. A 913, 28 (2019).

[15] A. Gover, F. V. Hartemann, G. P. Le Sage, N. C. Luhmann, R.S. Zhang, and C. Pellegrini, Time and Frequency Domain Analysis of Superradiant Coherent Synchrotron Radiation in a Waveguide Free-Electron Laser, Phys. Rev. Lett. 72, 1192 (1994).

[16] M. Tecimer and L. Elias, Three-dimensional simulation of finite pulse effects in SASE via Lienard-Wiechert fields, Nucl. Instrum. Methods Phys. Res., Sect. A 393, 104 (1997).

[17] P. Schmüser, M. Dohlus, and J. Rossbach, Ultraviolet and Soft X-Ray Free-Electron Laser (Springer Tracts in Modern Physics, New York, 2008), Vol. 229.

[18] N. McLachlan, Theory and Application of Mathieu Functions (Oxford University Press, New York, 1947).

[19] M. Quattromini, M. Artioli, E. Di Palma, A. Petralia, and L. Giannessi, Focusing properties of linear undulators, Phys. Rev. ST Accel. Beams 15, 080704 (2012).

[20] T. C. Marshall, Free-electron lasers, (Macmillan Publishing Company, 1985), ISBN .

[21] A. T. G. Dattoli and A. Renieri, Lectures on the Free Electron Laser Theory and Related Topics (World Scientific, Singapore, 1993).

[22] J. D. Jackson, Classical Electrodynamics, 3rd ed. (Wiley, New York, 1999).

[23] E. W. Weisstein, Mathieu function from MathWorld-A Wolfram Web Resource. 